\title{
Estresse e Ajustamento Conjugal de Casais com Filho(a) com Síndrome de Down
}

\author{
Nara Liana Pereira-Silva* \\ Universidade Federal de Juiz de Fora, Juiz de Fora, MG, Brasil
}

\begin{abstract}
RESUMO
O presente estudo teve o objetivo de investigar estresse e ajustamento conjugal em 19 casais com um(a) filho(a) com síndrome de Down. As crianças tinham entre quatro e dez anos, sendo 11 do sexo masculino e 8 do sexo feminino. Utilizou-se o Questionário de Caracterização do Sistema Familiar, Dyadic Adjustment Scale em seu formato traduzido para a língua portuguesa do Brasil (Escala de Ajustamento Diádico) e o ISSL para adultos. As mães são mais acometidas pelo estresse $(n=11)$ do que os pais $(n=7)$. Todas as mães percebem seu relacionamento conjugal como ajustado e somente dois pais o percebem como desajustado. Não foram encontradas associações significativas entre escores da EAD, sexo e idade dos genitores; e tempo de convivência do casal.
\end{abstract}

Palavras-chave: estresse; relação conjugal; síndrome de Down; família.

\begin{abstract}
Stress and Marital Adjustment of Couples with Children with Down Syndrome

The present study aimed to investigate the stress and marital adjustment in 19 couples with a child with Down syndrome. The children were between four and ten years, 11 males and 8 females. We used the Family Characteristics Questionnaire, the Dyadic Adjustment Scale in its format translated into Portuguese of Brazil (Dyadic Adjustment Scale) and ISSL for adults. Mothers are more affected by stress $(\mathrm{n}=11)$ than fathers $(\mathrm{n}=7)$. All mothers perceive their marital relationship as adjusted and only two fathers perceive as inadequate. No significant associations were found between scores of DAS, sex and age of the parents, and the couple's time together.
\end{abstract}

Keywords: stress; marital relation; Down syndrome; family.

Estudar a família é uma tarefa difícil, tendo em vista a complexidade que a envolve. Trata-se de um grupo entendido de forma sistêmica, composto por subsistemas integrados e interdependentes, que estabelece uma relação bidirecional e de mútua influência com o contexto sócio-histórico-cultural no qual está inserida (Minuchin, 1988). Ela é, também, concebida como um dos primeiros contextos de socialização dos indivíduos em desenvolvimento, possuindo um papel fundamental para o entendimento do processo de desenvolvimento humano (Kreppner, 2000, 2003), especialmente em se tratando de crianças com deficiência.

No que se refere às relações familiares, não se pode negar o papel importante dos sentimentos, emoções e reações de todos os membros em relação a cada um dentro do grupo e, especialmente, direcionado à criança com deficiência. O tipo de reação emocional mais comumente medido em mães de crianças com deficiências é o estresse (Cramm \& Nieboer, 2011; Dabrowska \& Pisula, 2010; Hastings, Daley, \& Burns, 2006). Os estudos têm mostrado que mães de crianças com deficiência intelectual (DI) experienciam mais estresse que os pais nesse mesmo grupo e também que as mães de crianças com desenvolvimento típico (Dyson, 1997; Hastings et al., 2006; Hornby, 1995; Lamb \& Billings, 1997; Shapiro, Blacher, \& Lopez, 1998). No entanto, há dados que mostram níveis similares de estresse em mães e pais de crianças com deficiência intelectual (DI) em idade escolar (Dyson). 
Segundo Crnic, Arbona, Baker e Blacher (2009) o estresse não é um construto unidimensional, podendo ser definido de diferentes maneiras e, geralmente, a fonte ou o contexto estressor é o aspecto chave como unidade definidora do estresse. Há, portanto, estudos que descrevem o estresse geral da vida, o estresse parental, estresse em decorrência de ter um filho(a) com DI, mas poucos estudos têm incluído o pai (Crnic et al., 2009). Segundo estes autores, ao longo do tempo, os resultados das pesquisas têm mostrado que nem todo estressor tem impacto igual para toda pessoa (pai e mãe); e as demandas associadas à DI, por si só, não parecem ser estressantes, tal como as demandas associadas com problemas de comportamento da criança.

A literatura aponta que o estresse percebido pelos genitores de crianças com DI está associado aos seguintes fatores: temperamento da criança, isolamento e retirada social (Shapiro et al., 1998), status empregatício e satisfação conjugal (Hornby, 1995), à própria avaliação do genitor a respeito do funcionamento da família (Dyson, 1997), à satisfação com o apoio recebido dos profissionais (Dempsey, Keen, Pennell, O'Reilly, \& Neilands, 2009) e com o apoio recebido de familiares e não familiares/informal (Gerstein, Crnic, Blacher, \& Baker, 2009) e bem estar psicológico (Cramm \& Nieboer, 2011). Além desses fatores, Minnes (1998) afirma que os estudos sobre estresse em famílias com membros com deficiência podem ser agrupados segundo as características da criança, considerando a combinação de diferentes tipos de deficiências e o impacto da sobrecarga de cuidado com a criança. Estes aspectos são importantes, pois a severidade da deficiência da criança tem emergido como contribuidor para o estresse parental associado à dependência da criança, às necessidades de manejo, aos problemas de comportamento e à comunicação limitada. Verifica-se, portanto, que as características da criança, do ambiente e dos genitores têm desempenhado um papel importante na experiência de estresse parental (Hill \& Rose, 2009), implicando a multiplicidade de fatores que se associam.

No que se refere ao estresse de mães ao longo do tempo, os resultados de Most, Fidler, Laforce-Booth e Kelly (2006) com um grupo de mães de crianças com síndrome de Down (SD) e outro com mães de crianças com deficiência com etiologias mistas, revelaram que, em média, o estresse, na análise mista desses grupos, foi mais elevado no primeiro momento da coleta de dados, quando as crianças tinham entre 12 a 15 meses de idade e manteve-se inalterado ao longo do tempo, enquanto que o estresse no grupo de mães com filho com SD foi menor no primeiro momento da coleta de dados, mas aumentou de forma constante. Além disso, Most et al. (2006) apontam que o funcionamento cognitivo-linguístico mais avançado e níveis mais baixos de comportamentos desajustados das crianças, em todas as fases da pesquisa, foram associados com níveis mais baixos de estresse materno. Esse resultado aponta para as associações entre o desenvolvimento cognitivo-linguístico e problemas de comportamento e as mudanças no estresse materno.

Os resultados de Gerstein et al. (2009) sugerem de fato que a experiência de estresse ao longo do tempo é determinada de forma complexa pela variedade de fatores que operam para predizer tanto o nível de estresse como a trajetória do estresse durante a primeira infância, ou ambos. Em relação às diferenças e similaridades do estresse parental, Gerstein et al. (2009) ressaltam que a experiência diária de estresse de pais e mães difere significativamente não somente no nível, mas também em relação ao grau em que o estresse é vivenciado ao longo do tempo. Segundo esses autores, não somente a mãe tem estresse mais elevado que o pai, mas o estresse dela aumenta significativamente no período pré-escolar, enquanto o estresse diário do pai não.

Em se tratando de estudos nacionais, Cherubini, Bosa e Bandeira (2008) investigaram o índice de estresse e o autoconceito em mães e pais de 15 crianças com a síndrome do X-Frágil, 15 com SD e 15 com desenvolvimento típico (DT). Houve menos ocorrência de estresse entre os genitores no grupo de crianças com DT. No caso dos genitores de crianças com SD, $86,7 \%$ dos pais $(n=13)$ e $53,3 \%$ das mães $(n=8)$ não apresentaram sintomas de estresse. A fase do estresse em que os genitores se encontravam foi de 'Resistência'. Os resultados não apontam para diferenças significativas quando se compararam os grupos em relação ao estresse, mas os resultados revelaram diferença significativa em autoconceito. Cherubini e cols. (2008) destacam que outros fatores podem ter influência no estresse, tal como a existência de suporte familiar e profissional nos grupos clínicos (X-Frágil e SD). Esses fatores indicaram a importância da rede social de apoio familiar e não familiar para o enfrentamento dos desafios destas famílias. Nesse estudo o instrumento aplicado para medir o estresse nos genitores foi o Inventário de Sintomas de Stress para Adultos de 
Lipp - ISSL (Lipp, 2000/2011). Nos estudos de Da Silva $(2007,2011)$ também foi utilizado o ISSL para avaliar o índice de estresse nos genitores de crianças com SD. Os resultados indicaram estresse mais elevado em mães do que em pais. Além disso, foi encontrada uma associação entre o estresse do pai e o estresse da mãe, indicando que quanto maior o estresse vivenciado pelo pai, maior o estresse da mãe, bem como, quanto menor estresse do pai menor o da mãe (Da Silva, 2007). Destaca-se que não foi encontrada associação entre o estresse e a presença da criança com SD (Da Silva, 2011).

Além do estresse, os genitores de crianças com DI têm uma sobrecarga adicional em vários aspectos de sua dinâmica individual e familiar, tais como no psicológico, no social, no financeiro e nas atividades de cuidado com a criança (Ali et al., 1994; Eisenhower \& Blacher, 2006; Shapiro et al., 1998). Essa sobrecarga pode estar relacionada aos sentimentos de ansiedade e incerteza, considerando as questões de sobrevivência da criança, o seu desenvolvimento, o cuidado em longo prazo, o próprio impacto desse cuidado sobre a vida pessoal dos genitores etc. As mães, por exemplo, mesmo quando estão empregadas, ainda suportam uma desproporcional quantidade de responsabilidades com os cuidados da criança que podem justificar, em parte, o senso de sobrecarga e restrição em suas vidas pessoais (Shapiro et al., 1998). Por outro lado, os pais dessas crianças sentem mais sobrecarga que os pais de crianças com DT (Rodrigue, Morgan, \& Geffken, 1992).

De acordo com a literatura e com a perspectiva sistêmica, portanto, a dinâmica e funcionamento das famílias com filho(a) com DI pode ter implicações em diferentes subsistemas familiares (Braz, Dessen, \& Pereira-Silva, 2005; Erel \& Burman, 1995; Kreppner, 2000) e, em particular, no conjugal (Norlin \& Broberg, 2012; Stoneman, Gavidia-Payne, \& Floyd 2006; Wieland \& Baker, 2010). O relacionamento conjugal tem sido apontado como um fator preponderante para a qualidade de vida das famílias, particularmente no que tange às relações que pais e mães mantêm com suas crianças.

Estudos sobre a qualidade do relacionamento conjugal de casais com filho com DI têm mostrado resultados controversos (Risdal \& Singer, 2004; Shapiro \& cols., 1998). A meta-análise conduzida por Risdal e Singer (2004) que comparou a qualidade conjugal de genitores de crianças com e sem deficiência recuperou estudos com resultados discrepantes; alguns relatam pior qualidade conjugal ou nenhuma diferença na qualidade do relacionamento entre o casal; outros apresentam níveis superiores de qualidade para casais com uma criança com deficiência. Dois estudos do final da década de 1980, Carr (1988) e Bristol, Gallagher e Schopler (1988), não encontraram diferenças significativas entre as famílias de crianças com e sem $\mathrm{DI}$, especialmente a $\mathrm{SD}$, no tocante à qualidade da relação marital, coesão e satisfação conjugal. Em famílias de crianças com DI tem-se encontrado níveis de satisfação conjugal considerados bons (Da Silva, 2011), isto é, iguais ou superiores à média (Stoneman et al., 2006). No estudo de Da Silva (2011) os casais que tiveram níveis mais altos de satisfação conjugal apresentaram, também, baixos níveis de estresse relacionados à presença da criança, ao estresse geral e sintomas de depressão.

O estudo longitudinal de Wieland e Baker (2010) com 132 famílias, sendo 48 com uma criança com DI e 84 com crianças com DT, não observou diferenças significativas na média da qualidade conjugal e nos escores de apoio do cônjuge entre os grupos investigados. A variância nos escores de qualidade conjugal foi significativamente maior para os casais com filho(a) com DI do que para aqueles com filho(a) com DT. Além disso, os resultados mostraram, também, que escores mais altos no domínio marital estavam relacionados com escores mais baixos de problemas de comportamento da criança. Norlin e Broberg (2012), ao pesquisarem uma amostra composta por crianças com e sem DI associada a problemas de comportamento, chegaram a conclusões similares às de Wieland e Baker, uma vez que a qualidade da relação conjugal não diferiu entre os grupos, mas a coparentalidade diferiu em nível de uma tendência. Estes resultados refletem a importância relativa do sistema de coparentalidade em situações de estresse relacionadas com a criança com DI. Segundo os autores, é provável que os desafios relacionados ao crescimento de uma criança com necessidades especiais e problemas de comportamento fortaleçam a capacidade dos genitores para cooperar e apoiar um ao outro, mas esse processo não necessariamente repercute em um relacionamento romântico.

É interessante destacar que, ao longo do tempo, a qualidade da relação conjugal tende a diminuir, quando o(a) filho(a) com DI atinge a pré-adolescência e adolescência, segundo os resultados de Richardson 
(2012). Entretanto, quando esses filhos estão próximos da vida adulta, os resultados de Richardson sugerem um aumento nos escores da qualidade conjugal para ambos os genitores. Para a autora, o declínio inicial da satisfação conjugal desses casais, provavelmente, representa uma diminuição moderada no grau de satisfação, mas não indica necessariamente uma transformação qualitativa de um casamento feliz para um casamento infeliz. Esse padrão é similar ao encontrado em famílias com filhos com DT, mostrando um declínio na qualidade do relacionamento conjugal quando os filhos estão na adolescência e, posteriormente, um aumento nos níveis dessa qualidade quando os filhos completam a fase de 'lançamento', ou seja, quando eles deixam o lar (Richardson, 2012).

Assim, considerando a escassez de pesquisas com famílias de pessoas com DI, especialmente com SD, no Brasil, e diante da necessidade de compreender mais profundamente a dinâmica dessas famílias, foi realizado um estudo com os seguintes objetivos: (1) identificar a presença de sintomas de estresse percebidos por pais e mães de crianças com SD e (2) caracterizar a qualidade das relações conjugais nessas famílias, a partir das dimensões de consenso, satisfação, coesão conjugal e expressão de afeto.

\section{MÉTODO}

\section{Participantes}

Participaram deste estudo 19 famílias compostas por mãe, pai e filhos tendo uma deles o diagnóstico de Síndrome de Down. Todos os membros da família moravam juntos na área urbana $(n=18)$ ou rural $(n=$ 1) de Juiz de Fora, Minas Gerais. Onze crianças eram do sexo masculino e oito do sexo feminino, com idades que variavam de quatro a dez anos, com média de 7 anos. A média da idade dos pais foi de 44,8 anos e das mães 42,8 anos. A média do tempo de convivência dos casais foi de 15,9 anos. As famílias tinham, em média, 2,26 filhos. A renda familiar teve uma média de 5,56 salários mínimos à época da coleta de dados. Foram relatadas diversas ocupações dos pais, sendo profissional liberal $(n=5)$ e serviços técnicos em geral $(\mathrm{n}=5)$ as mais frequentemente relatadas. Somente um pai estava desempregado, um aposentado e as demais ocupações dos pais eram: militar $(n=1)$, serviços de comércio e venda $(n=1)$, serviços operacionais $(n=1)$, outras $(n=4)$. Já as mães apresentavam um panorama diferente quanto à ocupação, uma vez que oito não trabalhavam fora de casa. Assim, as ocupações relatadas foram pelas mães foram: profissional liberal $(n=6)$, professora $(n=2)$, serviços de comércio e venda $(\mathrm{n}=1)$, auxiliar de cozinha $(\mathrm{n}=1)$, outros $(\mathrm{n}=1)$.

\section{Instrumentos}

Para a coleta de dados, utilizaram-se o Questionário de Caracterização do Sistema Familiar, o Inventário de Sintomas de Stress para Adultos de Lipp - ISSL e a Dyadic Adjustment Scale na versão traduzida para o português.

O Questionário de Caracterização do Sistema Familiar (Dessen, 2009) é um instrumento que tem o objetivo de coletar informações sobre idade e escolaridade dos genitores e filhos, renda familiar, ocupação dos genitores, estado civil, contatos sociais da família, dados sobre a rede social de apoio, identificação dos responsáveis pelas tarefas rotineiras desenvolvidas com a criança, as atividades de lazer desenvolvidas pela família dentre outros dados do contexto familiar, tais como fatores de risco que envolvem a família.

O Inventário de Sintomas de Stress para Adultos de Lipp - ISSL (Lipp, 2000) mede a frequência de sintomas de estresse, o tipo de sintoma existente (se somático ou psicológico) e a fase de estresse em que o respondente se encontra. Apresenta um modelo quadrifásico, cuja primeira fase é a do alerta (o organismo prepara-se para a preservação da própria vida); seguida pela fase de resistência (o organismo tenta uma adaptação). A terceira fase é a de quase exaustão (enfraquecimento da pessoa que não consegue mais adaptar-se ou resistir ao estressor) e, por último, a fase de exaustão (o organismo exaure sua reserva de energia adaptativa e aparecem as doenças mais sérias). Quanto maior a pontuação no ISSL, maior a probabilidade de alcançar sintomas significativos de estresse e mais avançada a fase deste.

A versão utilizada para o presente estudo da Dyadic Adjustment Scale - DAS (Spanier, 1976) foi em seu formato traduzido para a língua portuguesa do Brasil (Escala de Ajustamento Diádico - EAD), cuja tradução utilizada no presente trabalho foi realizada pelo Laboratório de Desenvolvimento Familiar/UnB. Ela abrange quatro dimensões do ajustamento conjugal: consenso, satisfação, coesão e expressão de afeto. No tocante ao consenso diádico, esta dimensão referese à percepção individual de aspectos do relaciona- 
mento e do nível de concordância do casal sobre uma variedade de questões básicas, tais como: financeiras, atividades de lazer, religiosas, amizades, de convencionalidade, de filosofia de vida, de negócios com parentes, de metas e objetivos, de tempo alocado, de participação na tomada de decisão, de participação nas tarefas domésticas, de decisões sobre as carreiras profissionais. A satisfação diádica examina as percepções de cada um a respeito de situações que envolvem a possibilidade do divórcio e/ou separação, da saída de casa, do arrependimento, do bem-estar, da confiança, da frequência de emissão de beijo, da questão da felicidade e do compromisso com o relacionamento. A coesão mede o grau de compartilhamento emocional do casal, bem como as percepções individuais relativas ao engajamento mútuo em interesses externos, à criação de ideias, à diversão conjunta, à discussão tranquila e ao trabalho conjunto em projetos. A expressão de afeto entre o casal refere-se às emissões de comportamentos afetivos de um para o outro.

\section{Procedimentos}

O presente estudo foi aprovado pelo Comitê de Ética em Pesquisa (CEP) da Universidade Federal de Juiz de Fora, sob o número 355. A Secretaria de Educação de Juiz de Fora disponibilizou uma lista de escolas, tendo sido contatadas 26 delas. Destas, 13 não tinham crianças com SD matriculadas. Duas instituições de educação especial também foram contatadas. Essas escolas foram visitadas para que pudessem fornecer o contato com as famílias de crianças com síndrome de Down para que fosse possível realizar o recrutamento e seleção das mesmas. Obteve-se o consentimento de 19 famílias para participar do estudo e a coleta de dados foi efetuada na residência das mesmas. O pai e a mãe assinaram o Termo de Consentimento Livre e Esclarecido na primeira visita à resi- dência da família. Além disso, a mãe respondeu ao Questionário de Caracterização do Sistema Familiar. A segunda visita ocorreu uma semana após a primeira, de acordo com a disponibilidade da família. Foram aplicados o Inventário de Sintomas de Stress para Adultos de Lipp e a Escala de Ajustamento Diádico com a mãe e com o pai, separadamente. Os instrumentos foram preenchidos por auxiliares de pesquisa.

Os dados foram tabulados em uma planilha eletrônica e submetidos a uma análise quantitativa, utilizando-se estatística descritiva (média, porcentagem, etc.). Destaca-se que, para classificar o ajustamento das díades a partir dos escores da EAD, foram adotados os critérios propostos por Spanier (1976). Logo, pontuações iguais ou inferiores a 101 foram categorizadas como desajustamento no relacionamento conjugal e, evidentemente, resultados superiores foram classificados como ajustamento conjugal. A correlação entre a EAD e as variáveis idade e tempo de convivência foi avaliada por meio do coeficiente de correlação Pearson. $\mathrm{O}$ Test $\mathrm{T}$ para amostras independentes foi utilizado para associar os escores dos padrões da EAD à variável sexo. O ISSL foi analisado segundo as normas constantes no Manual do próprio teste (Lipp, 2000).

\section{RESULTADOS}

Os resultados do Questionário de Caracterização do Sistema Familiar proporcionaram a identificação dos principais responsáveis pelos cuidados com o(a) filho(a) com SD e pelas atividades domésticas com a casa. As mães sozinhas ou com outros membros são as principais responsáveis pelas atividades de cuidado com o(a) filho(a) com SD. A Tabela 1 apresenta as frequências dos membros familiares, sozinhos ou não, que desenvolvem atividades com a criança com SD. 
Tabela 1

Membros Familiares Responsáveis pela Alimentação/Banho, Colocar para Dormir, Levar para a Escola e Levar para Atividades de Lazer

\begin{tabular}{|c|c|c|c|c|c|c|c|c|}
\hline \multirow{2}{*}{$\begin{array}{l}\text { Atividades/ } \\
\text { Tarefas }\end{array}$} & \multicolumn{3}{|c|}{ Membros sozinhos } & \multicolumn{4}{|c|}{ Membros que compartilham } & \multirow[b]{2}{*}{ Outros } \\
\hline & Pai & Mãe & Irmão & Pai e mãe & Mãe e irmãos & $\begin{array}{l}\text { Mãe e babá/ } \\
\text { empregada }\end{array}$ & Mãe, pai, irmãos & \\
\hline Alimentação/banho & --- & 11 & --- & 2 & --- & 2 & --- & 4 \\
\hline Colocar para dormir & 4 & 8 & --- & 5 & --- & --- & --- & 2 \\
\hline Levar para a escola & 2 & 8 & 2 & 2 & 2 & --- & --- & 3 \\
\hline Contar histórias & --- & 7 & 1 & 4 & 1 & --- & 2 & 4 \\
\hline $\begin{array}{l}\text { Levar para } \\
\text { atividades de lazer }\end{array}$ & 1 & 5 & 2 & 7 & --- & 1 & 2 & 1 \\
\hline
\end{tabular}

\section{Índice de estresse}

Quanto à presença de sintomas de estresse nos genitores $(\mathrm{N}=38$; pais $=19$; mães $=19)$, houve maior frequência desses sintomas em mães $(n=11)$ do que em pais $(n=7)$. Destes $(n=18), 9$ mães e 6 pais se encontravam na fase de Resistência e os demais na fase de Quase exaustão (mães - $n=2$; pai - $n=1$ ). Nenhum genitor encontrou-se na fase de Alerta ou na de Exaustão. No que se refere à sintomatologia, para o grupo de mães, foram mais frequentes os sintomas psicológicos $(n=7)$, seguidos pelos físicos $(n=2)$ e psicológicos e físicos $(n=2)$. Já, os pais apresentaram sintomas psicológicos $(n=3)$ e físicos $(n=3)$ e apenas um pai apresentou sintomas psicológicos e físicos simultaneamente. Os resultados apontam que as mães são mais acometidas pelo estresse $(n=11)$ do que os pais $(n=7)$. A Tabela 2 apresenta as frequências e percentuais de estresse, as fases e sintomas, segundo pais e mães.

Tabela 2

Frequências e Percentuais do Diagnóstico de Estresse, Fase e Tipo de Sintomatologia

\begin{tabular}{lcccc} 
Estresse & \multicolumn{2}{c}{ Pai } & \multicolumn{2}{c}{ Mãe } \\
Sem estresse & $\mathbf{n}$ & $\%$ & $\mathbf{n}$ & $\%$ \\
Com estresse & 12 & 63,2 & 8 & 42,1 \\
Fases do estresse & 7 & 36,8 & 11 & 57,9 \\
$\quad$ Alerta & & & & \\
$\quad$ Resistência & --- & ---- & ---- & --- \\
$\quad$ Quase exaustão & 6 & 85,7 & 9 & 81,8 \\
$\quad$ Exaustão & 1 & 14,3 & 2 & 18,5 \\
Sintomatologias & --- & --- & --- & --- \\
$\quad$ Físico & & & & \\
$\quad$ Psicológico & 3 & 42,8 & 2 & 18,2 \\
$\quad$ Psicológico-Físico & 3 & 42,8 & 7 & 63,6 \\
\hline
\end{tabular}

\section{Ajustamento conjugal}

Para a classificação do ajustamento conjugal, de acordo com Spanier (1976), indivíduos que obtiverem 101 pontos ou menos devem ser classificados como desajustados ou em sofrimento em seu relacionamento conjugal e aqueles que alcançarem 102 pontos ou mais, ajustados. Esse tipo de classificação também foi utilizado no Brasil em outros estudos sobre relacionamento conjugal (Hernandez, 2008; Magagnin et al., 2003). Verificou-se que a maioria das pessoas $(n=36)$ está em ajustamento, de acordo com o cálculo da pontuação na Escala de Ajustamento Diádico, considerando os resultados obtidos por Spanier (1976). De acordo com essa classificação, todas as mães $(n=19)$ e 
17 pais, no presente estudo, obtiveram pontuação correspondente à classificação de ajustamento conjugal. Apenas dois pais apresentaram percepção de díade desajustada. Não houve diferenças importantes entre os grupos, sendo que a média para as mães foi 120 pontos e para os pais, 118, acima da média na amostra original que é 101 pontos. A Tabela 3 mostra esses resultados.

Tabela 3

Ajustamento Conjugal Segundo Pais e Mães

\begin{tabular}{l|cccc} 
Dimensões do ajustamento & \multicolumn{2}{|c}{ Pai } & \multicolumn{2}{c}{ Mãe } \\
& $\mathbf{n}$ & $\%$ & $\mathbf{n}$ & $\%$ \\
\hline Ajustamento & 17 & 89 & 19 & 100 \\
Desajustamento & 2 & 11 & --- & --- \\
\hline
\end{tabular}

A Tabela 4 mostra a média e o desvio padrão em cada fator da EAD, segundo cada grupo de genitores.

\begin{tabular}{|c|c|c|c|c|}
\hline \multirow{2}{*}{ Fatores } & \multicolumn{2}{|c|}{ Pai } & \multicolumn{2}{|c|}{ Mãe } \\
\hline & $x$ & DP & $x$ & DP \\
\hline Consenso & 56,26 & 6,79 & 57,31 & 4,6 \\
\hline Satisfação & 35,2 & 3,13 & 35 & 2,33 \\
\hline Coesão & 16,10 & 4,3 & 17,52 & 3,23 \\
\hline Expressão de Afeto & 10,42 & 1,74 & 10,7 &, 80 \\
\hline Total & 118 & 9,98 & 120,5 & 7,8 \\
\hline
\end{tabular}

Observa-se que, em todos os fatores, os genitores apresentam médias similares, sendo que aquelas encontradas nos fatores Consenso, Coesão e Expressão de afeto são superiores às encontradas por Spanier (1976), as quais são 52,8; 11,8 e 7,8, respectivamente. Somente no fator Satisfação a média encontrada foi similar à amostra original, isto é, 35.

Foram realizadas análises correlacionais entre as variáveis demográficas e os escores obtidos na EAD, entretanto, não foram encontradas associações significativas ao associar os escores obtidos na $\mathrm{EAD}$ à variável sexo do genitor $[t(36,38)=-0,89 ; \mathrm{p}>0,05]$. Também não foram encontradas associações significativas entre os escores obtidos na EAD e a idade do genitor $(r=-0,16 ; \mathrm{p}>0,05)$, bem como entre os escores obtidos na EAD e tempo de convivência do casal $(r=-0,07 ; \mathrm{p}>0,05)$.

Associações entre o escore total de estresse e o escore obtido na EAD foram realizadas, tendo sido muito discrepantes com média $\mathrm{X}=11,71$ e o desvio padrão, também, muito grande $\mathrm{DP}=8,70$, assim não foi encontrada associação significativa entre os escores do ISSL com a EAD.

\section{DISCUSSÃO}

Nas famílias participantes do presente estudo, as mães são as maiores responsáveis pelos cuidados (dar banho, alimentação etc.) e atividades diárias com a criança com síndrome de Down, tais como, contar histórias, colocar para dormir, levar para a escola e levar para atividades de lazer. Mesmo nas famílias com nível sócio econômico mais elevado, as mães também são as maiores responsáveis. Esse resultado é coerente com aquele encontrado na literatura, em que as mães se sobrecarregam com as atividades com os filhos com deficiência (Ali et al., 1994; Da Silva, 2011) mais do que outros membros da família. Os pais sozinhos, nessas famílias, realizam poucas atividades com o(a) filho(a), entretanto, observa-se a inserção deles em várias tarefas, tais como, colocar a criança para dormir, contar histórias e levá-la para atividades de lazer, mesmo que seja compartilhando com a mãe 
ou com outra pessoa. Apesar disso, não se pode afirmar que os pais não sejam participativos, mas, deve-se considerar que outros fatores influenciam nessa divisão de tarefas no lar, tais como o contexto cultural (Dessen, 2010), o contexto ocupacional, uma vez que em pelo menos oito famílias os pais são os únicos responsáveis pela renda familiar. A dinâmica das famílias brasileiras com filhos com deficiência intelectual poderia ser um tema importante para estudos futuros, uma vez que esse assunto pode ser um fator preditivo do estresse parental, o qual pode influenciar as relações familiares e, portanto, o desenvolvimento de seus membros com deficiência.

Devido às dificuldades em adequar as demandas com a criança com deficiência intelectual e a necessidade de cuidados extras, são comuns os altos níveis de estresse, principalmente, em mães (Ali et al., 1994; Dyson, 1997; Hornby, 1995; Lamb \& Billings, 1997; Shapiro \& cols., 1998; Sloper, Knussen, Turner, \& Cunningham, 1991). Os dados do presente estudo tendem a corroborar esses achados. As mães (mais de $50 \%$ ) são as mais acometidas pelo estresse (pais= $36,8 \%$ ), no entanto, não há casos de estresse em fase de Exaustão, estando a maioria dos genitores na fase de Resistência. Esses resultados são semelhantes a outros encontrados no Brasil, como em Cherubini e cols. (2008) e Da Silva $(2007,2011)$. Apesar de poucos genitores estarem na fase de Quase exaustão $(n=3)$ ressalta-se que os mesmos foram orientados, no momento de devolução dos resultados da pesquisa, quanto aos sintomas de estresse e seus riscos. É importante destacar que o instrumento utilizado para medir o estresse nos genitores, ISSL de Lipp, não se refere ao estresse parental propriamente, mas sim, ao estresse geral. Assim, não se pode afirmar necessariamente que o estresse avaliado seja em função do desempenho do papel de pai e mãe. Não há, ainda, em nosso país um instrumento padronizado e validado para medir esse tipo de estresse, tal como o Parental Stress Index (Abidin, 1995).

No que se refere ao ajustamento conjugal, os resultados apontam para relações conjugais caracterizadas por coesão, satisfação, consenso e expressão de afeto, indicando índice de ajustamento diádico superior à média. Esse resultado corrobora estudos anteriores (Da Silva, 2011; Stoneman et al., 2006) quanto aos bons índices de qualidade do relacionamento entre casais que têm filho(a) com SD. Entretanto, ressaltase a necessidade de estudar esse assunto com uma quantidade maior de participantes, o que permitiria a realização de associações mais robustas estatisticamente, especialmente, considerando o fato de não terem sido encontradas associações entre os escores da EAD e a idade, o sexo e o tempo de convivência do casal.

É interessante destacar que, apesar de 18 genitores apresentarem sintomas de estresse, os resultados indicam satisfação conjugal, demonstrando, provavelmente, que a sobrecarga de cuidados parece não ter implicações importantes nas relações desenvolvidas no subsistema conjugal. O resultado de não haver associação entre os escores de estresse e os da EAD, talvez, indique que o estresse não esteja associado à presença da criança, necessariamente, conforme resultados já encontrados por Da Silva (2011). Essas associações poderiam ser assunto para estudos futuros. Assim, mesmo em presença de estresse parece haver uma tendência à satisfação conjugal nessas famílias. Esse fato pode ser comprovado ainda pela análise da questão 31 da EAD sobre o 'grau de felicidade' com o relacionamento, em que é solicitado à pessoa que 'circule o número que melhor descreve o grau de felicidade (com o relacionamento), considerando todos os aspectos do seu relacionamento'. Nessa questão, as respostas variavam entre: extremamente infeliz, bastante infeliz, um pouco infeliz, feliz, muito feliz, extremamente feliz e perfeito, sendo feliz o grau de felicidade da maioria dos casamentos. No presente estudo, não houve diferenças importantes entre pais e mães das crianças com SD, uma vez que apenas um pai percebe seu casamento como 'um pouco infeliz' e todos os outros genitores, pais e mães, avaliaram seus relacionamentos entre feliz e perfeito, tendo uma tendência de pais que avaliaram como muito feliz $(n=7)$ e mães que percebem como feliz $(n=8)$ ou muito feliz $(n=7)$.

Embora o presente estudo não tenha investigado a associação entre a relação conjugal e a parentalidade, destaca-se a importância desse tipo de investigação para maior aprofundamento nas questões que envolvem o funcionamento familiar, especialmente comparando tipos de famílias, no Brasil. O estudo longitudinal de Richardson (2012) sobre o ajustamento de casais com filho com DI indicou que, quando os filhos estavam na adolescência, a qualidade do relacionamento tendia a diminuir. Na presente investigação, os filhos tinham idades que variaram entre 4 e 10 anos, indicando que, provavelmente, nessas famílias o relacionamento conjugal parece não ter sido afetado pela 
idade das crianças, conforme indicam os resultados de Richardson. Entretanto, é importante destacar que, ocorridos quatro anos ou mais do nascimento do filho com SD, é possível considerar que o grupo já tenha conseguido reestabelecer o equilíbrio, conforme apontam, por exemplo, Carter e McGoldrick (1995), Dessen (1997). Além disso, os casais tinham, em média, mais de 15 anos de convivência. Sugerem-se estudos futuros que focalizem os momentos de transição familiar, bem como as associações entre a conjugalidade e a parentalidade em famílias com filho(a) com SD. Destaca-se também a importância de investigações ao longo do tempo, ou quando os filhos estão em diferentes momentos do curso de vida, isto é, adolescência, vida adulta etc., uma vez que há escassez de estudos considerando essas etapas, especialmente no Brasil (Oliveira, 2013).

\section{REFERÊNCIAS}

Abidin, R. R. (1995). Parenting stress index. Odessa, FL: Psychological Assessement Resources.

Ali, M. R., Al-Shatti, A., Khaleque, A., Rahman, A., Ali, S. M., \& Ahmed, R. U. (1994). Parents of mentally retarded children: Personality characteristics and psychological problems. Social Behavior and Personality, 22, 41-52.

Braz; M. P.; Dessen, M. A., \& Pereira-Silva, N. L. (2005). Relações conjugais e parentais: uma comparação entre famílias de classes sociais baixa e média. Psicologia: Reflexão e Crítica, 18, 151 - 161.

Bristol, M. M., Gallagher, J. J., \& Schopler, E. (1988). Mothers and fathers of young developmentally disabled and nondisabled boys: Adaptation and spousal support. Developmental Psychology, 24, 441-451.

Carr, J. (1988). Six weeks to twenty-one years old: A longitudinal study of children with Down's syndrome and their families. Journal of Child Psychology and Psychiatry and Allied Disciplines, 29, 407-431.

Carter, B. \& McGoldrick, M. (1995). As mudanças no ciclo de vida familiar. Porto Alegre: Artes Médicas.

Cherubini, Z. A., Bosa, C. A., \& Bandeira, D. R. (2008). Estresse e autoconceito em pais e mães de crianças com a síndrome do X frágil. Psicologia: Reflexão e Crítica, $21,409-417$.

Cramm, J. M. \& Nieboer, A. P. (2011). Psychological wellbeing of caregivers of children with intellectual disabilities: Using parental stress as a mediating factor. Journal of Intellectual Disabilities, 15, 101-113.

Crnic, K., Arbona, A. P., Baker, B., \& Blacher, J. (2009). Mothers and fathers together: contrast in parenting across preschool to early school age in children with de- velopmental delays. Em L. M. Glidden \& M. M. Seltzer (Orgs.), International review of research in mental retardation: families, Vol. 37 (pp. 3-30). San Diego: Elsevier.

Da Silva, N. C. B. (2011). Intervenção Domiciliar e Envolvimento Paterno: Efeitos em Família de Crianças com Síndrome de Down. Tese de Doutorado, Universidade Federal de São Carlos, São Carlos-SP.

Da Silva, N. C. B. (2007). Contextos familiares de crianças com síndrome de Down: interação e envolvimento paterno e materno. Dissertação de Mestrado, Universidade Federal de São Carlos, São Carlos-SP.

Dessen, M. A. (2010). Estudando a família em desenvolvimento: desafios conceituais e teóricos. Psicologia: Ciência e Profissão, 30, 202-219.

Dessen, M. A. (2009). Questionário de categorização do sistema familiar. In: L. Weber \& M. A. Dessen (Orgs.), Pesquisando a família: instrumentos para coleta e análise de dados (pp. 119-131). Curitiba: Juruá.

Dessen, M. A. (1997). Desenvolvimento familiar: transição de um sistema triádico para poliádico. Temas em Psicologia, 3, 51-61.

Dabrowska, A. \& Pisula, E. (2010). Parenting stress and coping styles in mothers and fathers of pre-school children with autism and Down syndrome. Journal of Intellectual Disability Research, 54, 266-280.

Dempsey, I., Keen, D., Pennell, D., O’Reilly, J., Neilands, J. (2009). Parent stress, parenting competence and family-centered support to young children with an intellectual or developmental disability. Research in Developmental Disabilities, 30, 558-566.

Dyson, L. L. (1997). Fathers and mothers of school-age children with developmental disabilities: Parental stress, family functioning, and social support. American Journal on Mental Retardation, 102, 267-279.

Eisenhower, A. \& Blacher, J. (2006). Mothers of young adults with intellectual disability: multiple roles, ethnicity and well-being. Journal of Intellectual Disability Research, 50, 905-916.

Erel, O., \& Burman, B. (1995). Interelatedness of marital relations and parent-child relations: A meta-analytic review. Psychological Bulletin, 118, 108-132.

Gerstein, E. D., Crnic, K. A., Blacher, J., \& Baker, B. L. (2009). Resilience and the course of daily parenting stress in families of young children with intellectual disabilities. Journal of Intellectual Disability Research, 53, 981-997.

Hastings, R. P., Daley, D., \& Burns, C. (2006). Maternal distress and expressed emotion: cross-sectional and longitudinal relationships with behavior problems of children with intellectual disabilities. American Journal on Mental Retardation, 11, 48-61. 
Hernandez, J.A.E. (2008). Avaliação estrutural da escala de ajustamento diádico. Psicologia em Estudo, 13, 593-601.

Hill, C. \& Rose, J. (2009). Parenting stress in mothers of adults with an intellectual disability: parental cognitions in relation to child characteristics and family support. Journal of Intellectual Disability Research, 53, 969-980.

Hornby, G. (1995). Effects on fathers of children with Down syndrome. Journal of Child and Family Studies, 4, 239-255.

Kreppner, K. (2000). The child and the family: Interdependence in developmental pathways. Psicologia: Teoria e Pesquisa, 16, 11-22.

Kreppner, K. (2003). Social relations and affective development in the first two years in family contexts. Em J. Valsiner \& K. J. Connolly (Orgs.), Handbook of developmental psychology (pp. 194-214). London: Sage.

Lamb, M.E. \& Billings, L.A.L. (1997). Fathers of children with special needs. Em M.E. Lamb (Org.), The role of the father in child development (pp. 179-190). New York: Wiley.

Lipp, M. N. (2000). Inventário de Sintomas de Stress para Adultos de Lipp. São Paulo: Casa do Psicólogo Livraria e Editora Ltda.

Magagnin, C., Körbes, J.M., Hernandez, J. A. E., Cafruni, S., Rodrigues, M. T., \& Zaperlon, M. (2003). Da conjugalidade à parentalidade: gravidez, ajustamento e satisfação conjugal. Aletheia, 17/18, 41-52.

Minnes, P. (1998). Mental retardation: The impact upon the family. Em J. A. Burack, R. M. Hodapp \& E. Zigler (Orgs.), Handbook of mental retardation and development (pp. 693-712). Cambridge: Cambridge University Press.

Minuchin, P. (1988). Relationships within the family: A systems perspective on development. Em R. A. Hinde \& J. Stevenson-Hinde (Orgs.), Relationships within families: Mutual influences. Oxford: Clarendon Press.

Most, D. E., Fidler, D. J., Laforce-Booth, C., \& Kelly, J. (2006). Stress trajectories in mothers of young children with Down syndrome. Journal of Intellectual Disability Research, 50, 501-514.

Norlin, D. \& Broberg, M. (2012). Parents of children with and without intellectual disability: couple relationship and individual well-being. Journal of Intellectual Disability Research, 1-15.
Oliveira, L. D. (2013). Famílias de adolescentes com deficiência intelectual: estresse, estratégias de enfrentamento e apoio social. Dissertação de mestrado, Universidade Federal de Juiz de Fora, Juiz de Fora-MG.

Richardson, S. S. (2012). Developmental Trajectories of Marriage, Coparenting, and Parenting Stress for Parents of Adolescents and Young Adults with Intellectual Disability. Retirado em 16 de maio de 2012 de http://digitalarchive.gsu.edu/psych_diss/100

Risdal, D.; Singer, G. H. (2004). Marital adjustment in parents of children with disabilities: a historical review and meta-analysis. Research and Practice for Persons with Severe Disabilities, 29, 95-103.

Rodrigue, J. R., Morgan, S. B., \& Geffken, G. R. (1992). Psychosocial adaptation of fathers of children with autism, Down syndrome and normal development. Journal of Autism and Development Disorders, 22, 249-263.

Shapiro, J., Blacher, J., \& Lopez, S. R. (1998). Maternal reactions to children with mental retardation. Em J.A. Burack, R. M. Hodapp \& E. Zigler (Orgs.), Handbook of mental retardation and development (pp. 606-636). Cambridge: Cambridge University Press.

Sloper, P., Knussen, C., Turner, S., \& Cunningham, C. (1991). Factors related to stress and satisfaction with life in families of children with Down's syndrome. Journal of Child Psychology and Psychiatry and Allied Disciplines, 32, 655-676.

Spanier, G. B. (1976). Measuring dyadic adjustment: New scales for assessing the quality of marriage and similar dyads. Journal of Marriage and the Family, 38, 15-28.

Stoneman, Z., Gavidia-Payne, S., \& Floyd, K. (2006). Marital Adjustment in families of young children with disabilities: Associations with daily hassles and problemfocused coping. American Journal on Mental Retardation, 111, 1-14.

Wieland, N. \& Baker, B. L. (2010). The role of marital quality and spousal support in behaviour problems of children with and without intellectual disability. Journal of Intellectual Disability Research, 54, 620-633.
Recebido em: 16/07/2013

Última Revisão em: 10/12/2014 Aceito em: 20/05/2015 